\title{
Vorwort Heft 4-2011
}

\author{
Hans-Christoph Grunau
}

Online publiziert: 12 . Oktober 2011

(C) Vieweg+Teubner und Deutsche Mathematiker-Vereinigung 2011

Am 3. Juni 2010, neun Tage vor seinem 73. Geburtstag, verstarb in Paris Vladimir Igorevich Arnold. Begraben wurde er in Moskau, der Stadt, in der er die meiste Zeit gelebt und gearbeitet hat; seit 1993 war er allerdings zugleich auch an der Dauphine in Paris tätig. Lassen sich - nicht zuletzt bedingt durch Reisebeschränkungen seitens der sowjetischen Behörden - die Aufenthaltsorte Arnolds relativ genau eingrenzen, so gelingt dieses hinsichtlich seines Schaffens und seiner Wirksamkeit nun überhaupt nicht. Zwar konzentrieren sich Leonid Polterovich und Inna Scherbak - langjährige Teilnehmer an Arnolds Seminaren - im vorliegenden Nachruf auf Hamiltonsche Dynamik, symplektische Topologie und Singularitätentheorie, beigetragen hat er aber in mehr als 20 Büchern (die teilweise in mehreren Auflagen erschienen und in verschiedene Sprachen übersetzt wurden) und mehr als 300 Arbeiten zu zahlreichen Gebieten der Mathematik von der Algebraischen Geometrie über Differentialgleichungen, globale Analysis, Hydromechanik und statistische Mechanik hin zur Zahlentheorie, um nur einige zu nennen. Den größten Anteil an Arnolds Bekanntheit haben vielleicht seine Durchbrüche in der KAM-Theorie, einem grundlegenden Resultat aus der Theorie Hamiltonscher Systeme zur Existenz quasiperiodischer Lösungen, das nach Kolmogorov, ihm selbst und Moser benannt ist. Im Nachruf findet sich auch eine lange Liste der Arnold zuerkannten Preise, eine Nominierung für die Fieldsmedaille 1974 wurde offenbar nach Intervention der Sowjetunion nicht weiter verfolgt.

Die Buchbesprechungen diskutieren Neuerscheinungen zu der zeitabhängigen von Kármánschen Differentialgleichung für dünne elastische Platten sowie aus dem Bereich der mathematischen Statistik und hier insbesondere zur Fehleranalyse statisti-

\footnotetext{
H.-Ch. Grunau ( $\bowtie)$

Institut für Analysis und Numerik, Fakultät für Mathematik, Otto-von-Guericke-Universität, Postfach 4120, 39016 Magdeburg, Deutschland

e-mail: hans-christoph.grunau@ovgu.de
} 
scher Schätzverfahren. Schließlich wird ein Buch zum Wechselspiel zwischen zellulären Automaten, Gruppen- und Ringtheorie vorgestellt, grundlegende Konzepte gehen hier auf das Werk John von Neumanns zurück. 